\title{
The tumour-promoting receptor tyrosine kinase, EphB4, regulates expression of Integrin- $\beta 8$ in prostate cancer cells
}

\author{
Inga Mertens-Walker ${ }^{1,2}$, Bruno C Fernandini', Mohanan SN Maharaj ${ }^{1}$, Anja Rockstroh ${ }^{2}$, Colleen C Nelson ${ }^{1,2}$, \\ Adrian C Herington ${ }^{1,2}$ and Sally-Anne Stephenson ${ }^{1,2^{*}}$
}

\begin{abstract}
Background: The EphB4 receptor tyrosine kinase is overexpressed in many cancers including prostate cancer. The molecular mechanisms by which this ephrin receptor influences cancer progression are complex as there are tumor-promoting ligand-independent mechanisms in place as well as ligand-dependent tumor suppressive pathways.

Methods: We employed transient knockdown of EPHB4 in prostate cancer cells, coupled with gene microarray analysis, to identify genes that were regulated by EPHB4 and may represent linked tumor-promoting factors. We validated target genes using qRT-PCR and employed functional assays to determine their role in prostate cancer migration and invasion.

Results: We discovered that over 500 genes were deregulated upon EPHB4 siRNA knockdown, with integrin $\beta 8$ (ITGB8) being the top hit (29-fold down-regulated compared to negative non-silencing siRNA). Gene ontology analysis found that the process of cell adhesion was highly deregulated and two other integrin genes, ITGA3 and ITGA10, were also differentially expressed. In parallel, we also discovered that over-expression of EPHB4 led to a concomitant increase in ITGB8 expression. In silico analysis of a prostate cancer progression microarray publically available in the Oncomine database showed that both EPHB4 and ITGB8 are highly expressed in prostatic intraepithelial neoplasia, the precursor to prostate cancer. Knockdown of ITGB8 in PC-3 and 22Rv1 prostate cancer cells in vitro resulted in significant reduction of cell migration and invasion.
\end{abstract}

Conclusions: These results reveal that EphB4 regulates integrin $\beta 8$ expression and that integrin $\beta 8$ plays a hitherto unrecognized role in the motility of prostate cancer cells and thus targeting integrin $\beta 8$ may be a new treatment strategy for prostate cancer.

Keywords: EphB4, Prostate cancer, Integrin- $\beta 8$

\section{Background}

Erythropoietin-producing hepatocellular (Eph) Type-B receptor 4 (EphB4) is part of the largest family of membrane-bound receptor tyrosine kinases (RTK) which consists of 14 different receptors which are classed as EphA or EphB. Their ligands, the ephrins, are also cell membrane-bound, either via glycosylphosphatidylinositol (GPI)-linkage (ephrin-A ligands) or transmembraneembedded (ephrin-B ligands). Interaction between Eph

\footnotetext{
* Correspondence: s.stephenson@qut.edu.au

${ }^{1}$ Institute of Health and Biomedical Innovation, Queensland University of

Technology, Translational Research Institute, 37 Kent Street, Woolloongabba, Queensland 4102, Australia

${ }^{2}$ Australian Prostate Cancer Research Centre - Queensland, Princess Alexandra Hospital, Woolloongabba, Queensland 4102, Australia
}

receptors and their ligands normally takes place in trans through the binding of 2 ligands on one cell to 2 receptors on an adjacent cell forming a heterotetramer that is the basic complex required for signaling. EphB4 plays an important role in cell signaling and is also involved in regulating cell morphology, adhesion, migration and invasion through modification of the cell's actin cytoskeleton and by influencing the actions of integrins [1]. Moreover, depending on the cell-environment conditions, EphB4 demonstrates the ability to be both a tumor promoter, when over-expressed and in the absence of stimulation by its sole cognate ligand, ephrin-B2, as well as a tumor suppressor stimulated by ephrin-B2 [2-6]. EphB4 is overexpressed in $66 \%$ of prostate cancer 
clinical samples and has been implicated in prostate cancer development and progression [2,7]. It has been shown using targeted siRNA sequences that knockdown of EphB4 in prostate cancer causes a significant reduction in cell motility in vitro and tumor growth in vivo [5]. However, the mechanisms by which removal of EphB4 exerts these effects are largely unknown. To date, no study has investigated the broader consequences on gene expression of siRNA-mediated knockdown of EPHB4 in prostate cancer. Therefore, we sought to determine the genome-wide changes upon transient knockdown of EPHB4 in a ligand-independent context in the prostate cancer cell line LNCaP.

Through gene expression analysis following EPHB4 knockdown, validation of the microarray data, and EphB4 over-expression, we have determined that EphB4 regulates the expression of integrin $\beta 8$ in prostate cancer cell lines.

\section{Methods}

\section{Cell culture}

All cell lines were purchased from the American Type Culture Collection (Manassas, VA). LNCaP, PC3 and 22Rv1 prostate cancer cells were cultured in RPMI 1640 (Life Technologies, Mulgrave, VIC, Australia) supplemented with $10 \%$ fetal calf serum (FCS). EphB4 overexpressing stable 22Rv1 cell lines, together with vector-only (VO) and parental $22 \mathrm{Rv} 1$ cells, were generated as described previously [2].

\section{siRNA transfection}

Lipofectamine 2000 (Life Technologies) was used to transiently transfect LNCaP cells with $10 \mathrm{nM}$ of EPHB4 siRNAs (SI00288589, SI04435053; Qiagen, Chadstone, VIC, Australia) or PC3 and 22Rv1 cells with $100 \mathrm{nM}$ of ITGB8 siRNAs (SI00034454, SI03066623, Qiagen). The AllStars non-silencing negative control siRNA (Qiagen) was used at the same concentration as gene-specific siRNAs for all experiments. After $48 \mathrm{~h}$, RNA from both the siRNA-treated cells and the EphB4 over-expressing cells was extracted using Trizol (Life Technologies).

\section{Microarray gene expression profiling}

Triplicate samples of EPHB4 siRNA knockdown and respective control siRNA transfected LNCaP cells were extracted for RNA and prepared for microarray profiling, which was performed on a custom Agilent $4 \times 180 \mathrm{~K}$ oligo array (VPCv3 ID:032034, GEO GPL16604, Agilent Technologies, Mulgrave, VIC, Australia). This microarray contains the Agilent 44 K (ID:014850) probe set incorporating human gene expression protein-coding probes as well as non-coding probes; with the probes targeting exonic regions, 3'UTRs, 5'UTRs, as well as intronic and intergenic regions [8]. RNA was isolated with
Trizol (Life Technologies), further purified using an RNeasy Mini Kit (Qiagen) with DNAse treatment according to the manufacturer's protocol. RNA samples were analyzed by a Bioanalyzer (Agilent) to ensure the RNA was of high quality. RNA (100 ng) from each group was amplified and labelled using the Low Input Quick Amp Labeling Kit (Agilent) and the protocol for One-Color Microarray-Based Gene Expression Analysis. The input RNA was reverse transcribed into cDNA, using an oligo-dT/T7-promoter primer which introduces a T7 promoter region. The subsequent in vitro transcription uses a T7 RNA polymerase, which simultaneously amplifies target material into cRNA and incorporates cyanine 3-labeled CTP. cDNA synthesis and in vitro transcription were both performed at $40^{\circ} \mathrm{C}$ for $2 \mathrm{~h}$. The labelled cRNA was then purified with Qiagen's RNeasy mini-spin columns and quantified using a Nanodrop1000 (Thermo Scientific, Waltham, MA, USA). cRNA $(1650 \mathrm{ng})$ from each sample was loaded onto the $4 \times 180$ $\mathrm{K}$ custom microarray and allowed to hybridize at $65^{\circ} \mathrm{C}$ for $17 \mathrm{~h}$. The arrays were scanned using an Agilent Microarray Scanner G2565CA.

\section{Microarray data analysis}

The microarray data were processed with Agilent Feature Extraction Software (v10.7). A quantile between-array normalization was applied and differential expression was determined using an unpaired T-test, asymptotic p-value and a 2-fold cut-off (GeneSpring GX11, Agilent Technologies). The gene expression levels are presented as fold change. Genes that were significantly different between two groups were identified with a p value of $<=0.05$, and an average fold change of $>=2$.

Normalized gene expression data of the experiment are Minimum Information About a Microarray Experiment (MIAME) and have been submitted to Gene Expression Omnibus (GEO) with the accession number GSE61800.

\section{Quantitative real-time PCR}

Extracted RNA was reverse transcribed using Superscript III reverse transcriptase according to the manufacturer's instructions (Life Technologies). Quantitative RT-PCR for EPHB4 expression was performed in triplicate using a TaqMan Gene Expression Assay (EPHB4: Hs00174752_m1 Life Technologies) and TaqMan Universal PCR Master Mix, No AmpErase UNG (Life Technologies) on a Rotor-Gene 6000 (Qiagen). The endogenous reference gene hydroxymethylbilane synthase (HMBS: Hs00609297_m1) was used for normalization and results are expressed as the EPHB4:HMBS ratio. For integrin gene expression analysis the SYBR Master Mix (Life Technologies) was used with GAPDH as a housekeeping gene. Primer sequences are listed in Table 1. 
Table 1 Primer sequences for semi-quantitative RT-PCR and qRT-PCR

\begin{tabular}{|c|c|c|c|}
\hline Gene & Forward primer $5^{\prime}$ to $3^{\prime}$ & Reverse primer $5^{\prime}$ to $3^{\prime}$ & Product size (bp) \\
\hline ITGA3 & AGCGCTACCTGCTCCTGGCT & GGGCAGTGAGTGGGCACAGG & 99 \\
\hline ITGA10 & CTGACAGGTCTCTGCTCCCCC & CCATCGCTGTCCACCCCCAA & 120 \\
\hline ITGB8 & TGTGTGCTGGGCATGGAGAGTGT & CAGTGCTGGGCTGCTGCTGAA & 100 \\
\hline ITGAV & CATCTGTGAGGTCGAAACAGG & TGGAGCATACTCAACAGTCTTTG & 137 \\
\hline EPHB4 & TCAATGTCACCACTGACCGAGAGGTA & GGTATTTGACCTCGTAGTCCAGCACA & 141 \\
\hline GAPDH & CTGCACCACCAACTGCTTAG & GTCTTCTGGGTGGCAGTGAT & 108 \\
\hline
\end{tabular}

\section{Semi-quantitative RT-PCR}

RT-PCR analysis was carried out using standard conditions with primers as shown in Table 1). PCR conditions were as follows: initial denaturation $94^{\circ} \mathrm{C}$ for $15 \mathrm{~min}$ then $94^{\circ} \mathrm{C}$ for $30 \mathrm{~s} ; 60^{\circ} \mathrm{C}$ for $30 \mathrm{~s} ; 72^{\circ} \mathrm{C}$ for $30 \mathrm{~s}$ repeated 35 times, final elongation $72^{\circ} \mathrm{C}$ for $10 \mathrm{~min}$. PCR products were analyzed on a $2 \%$ Tris-acetate agarose gel containing $0.01 \%$ ethidium bromide and photographed using a Gel doc system (Syngene, MD, USA).

\section{SDS-PAGE and western blotting}

Cells were lysed with ice-cold RIPA buffer (50 mM Tris $\mathrm{pH} 7.4,1 \%$ Triton X-100, 0.5\% sodium deoxycholate, $150 \mathrm{mM} \mathrm{NaCl}, 2 \mathrm{mM}$ EDTA, $1 \mathrm{mM}$ sodium orthovanadate, $1 \mathrm{mM}$ NaF, $1 \mathrm{mM}$ PMSF, $10 \mu \mathrm{g} / \mathrm{ml}$ aprotinin) supplemented with protease inhibitors (Complete Mini-EDTA-free tablets; Roche, Castle Hill, NSW, Australia). Protein lysates were mixed at $4^{\circ} \mathrm{C}$ for $15 \mathrm{~min}$, then insoluble proteins removed by centrifugation at $14,000 \mathrm{rpm}$. Protein concentrations were determined using the BCA Protein Assay Kit (Pierce, Rockford, IL, USA). Protein samples (20-50 $\mu \mathrm{g})$ were separated on SDS-PAGE gels (4\% stacking and 10\% separating) before proteins were transferred to a nitrocellulose membrane using a wet transfer system (Bio-Rad, Hercules, CA, USA) in transfer buffer (20\% methanol, $0.1 \mathrm{mM}$ Tris, $80 \mathrm{mM}$ glycine) at $100 \mathrm{~V}$ for $90 \mathrm{~min}$. After blocking with 5\% skim milk in TBST (Tris buffered saline with Tween-20:- $8 \mathrm{~g} / \mathrm{L} \mathrm{NaCl}, 3 \mathrm{~g} / \mathrm{L}$ Tris, $0.2 \mathrm{~g} / \mathrm{L} \mathrm{KCl}, 0.2 \%$ Tween-20, $\mathrm{pH}$ 7.4) for $1 \mathrm{~h}$, blots were probed with the primary antibody in 5\% BSA/ TBST (anti-EphB4 H-200, Santa Cruz Biotechnology, CA, USA) [9] or 5\% skim milk/TBST (anti-ITGB8 ab80673, Abcam, Melbourne, VIC, Australia) overnight at $4^{\circ} \mathrm{C}$ before incubation with horseradish peroxidaselabelled secondary antibody anti-rabbit IgG (SigmaAldrich, Castle Hill, NSW, Australia) in 5\% skim milk/TBST for $1 \mathrm{~h}$ at room temperature. Chemiluminescence was detected with Amersham ${ }^{\mathrm{Tm}}$ ECL Plus Chemiluminescence kit (GE Healthcare, Silverwater, NSW, Australia) following the manufacturer's recommendation, with a 10-30 sec exposure to SuperRX X-ray film (Fuji Film Corporation, Japan).

\section{Migration assay}

PC3 cells were seeded into 96-well Essen Bioscience ImageLock plates (Essen BioSciences, Ann Arbor, MI, USA) at $2 \times 10^{4}$ cells per well in four replicates and allowed to proliferate for $24 \mathrm{~h}$ until they had formed a complete monolayer. Cells were then transfected with ITGB8 siRNA and allowed to grow for a further $24 \mathrm{~h}$. The 96-well WoundMaker (Essen Bioscience) was then used to create a cell-free zone in the monolayer before the plates were placed in the IncuCyte ZOOM (Essen Bioscience). Migration into the cell free zone was determined after $24 \mathrm{~h}$ and quantified as relative wound density.

\section{Invasion assay}

Pre-coated growth-factor reduced Matrigel cell culture inserts (24-well, pore size $8 \mu \mathrm{m}$; BD Biosciences, San Jose, CA, USA) were seeded with serum-deprived $5 \times 10^{5}$ PC3 or 22Rv1 vector-only cells (22Rv1-VO) or 22Rv1 EphB4 over-expressing cells (22Rv1-B4) and transfected with $100 \mathrm{nM}$ ITGB8 siRNA or non-silencing AllStars siRNA (Qiagen) in 0.1\% FCS-containing medium. Medium containing $10 \%$ FCS was used as chemo-attractant. Cells were incubated for $22 \mathrm{~h}$ and cells that had not invaded were removed from the upper chamber using a cotton swab. Membranes were excised and mounted on glass slides with ProLong Gold Antifade containing 4, 6-diamidino-2phenylindole, dihydrochloride (DAPI) (Life Technologies) for visualization of the nuclei of cells that had invaded through the Matrigel to the underside of the membrane. Nuclei were counted in five random fields at $20 \mathrm{X}$ magnification using an Olympus epifluorescent microscope.

\section{Adhesion assay}

22Rv1-VO or -B4 cells, or their negative siRNA-control or ITGB8 siRNA counterparts ( 1 x $10^{5}$ cells), were seeded into triplicate wells in a Vitronectin pre-coated 96 well adhesion assay plate, and an adhesion assay was carried out according to the manufacturer's instructions (Merck Millipore, VIC, Australia).

\section{Statistics}

Statistical analysis was carried out using IBM SPSS software package by employing the ANOVA analysis 
followed by Fisher's least significant difference post hoc test or Student's t-test with $\mathrm{p}<0.05$ considered significant.

\section{Results \\ EPHB4 down-regulation results in differential gene expression in LNCaP cells}

In an effort to characterize the contribution of EphB4 to regulating gene expression in prostate cancer, endogenously EphB4-expressing LNCaP cells were transfected with EPHB4 specific siRNAs and compared to negative non-silencing siRNA cells using gene expression profiling. Quantitation of Western immunoblots using Image J confirmed knockdown of EPHB4 averaged $80.25 \pm 8.96 \%$. Using a 2 -fold cut off, 260 genes were up-regulated and 300 genes were found to be significantly down-regulated when EPHB4 siRNA transfectants were compared to the negative controls (Additional file 1: Figure S1). Among the top ten down-regulated genes were ITGB8 (29.4 fold), EIF4E3 (10.3 fold) and SASH1 (6.8 fold) (Table 2). On the other hand, the top ten up-regulated genes included $M Y O V I$ (5.7 fold), MMP12 (4.8 fold) and CYP11A1 (4.2 fold) (Table 3). Gene ontology screening highlighted the biological process of cell adhesion as being significantly de-regulated upon EPHB 4 knockdown (Table 4). Amongst the genes involved in cell adhesion were three integrin molecules that were deregulated (Table 4) including the top down-regulated gene, ITGB8. We therefore chose to further investigate the relationship between EphB4 and those three integrins ITGB8, ITGA3 and ITGA10.

Integrins are significantly de-regulated in response to changing EphB4 levels in prostate cancer cells

The microarray analysis revealed significant quantitative changes in ITGB8 and ITGA10 (down-regulated) and ITGA3 (up-regulated) (Figure 1A). To confirm these data we employed real-time PCR analysis on a different set of LNCaP transfectants. Upon EPHB4 knockdown, ITGB8 and ITGA10 were significantly down-regulated (Figure 1B). ITGA3 however, was unchanged (Figure 1B). Knockdown of EPHB4 with two different siRNAs also resulted in a reduction of integrin $\beta 8$ protein expression (Figure 1C), confirming the results seen at the gene level. Conversely, to investigate whether EphB4 overexpression would result in a parallel increase in ITGB8 expression, and to ensure that these data were not due to siRNA off-target effects, the expression of ITGB8 in stable 22Rv1-EphB4 over-expressing cells was also determined using qRT-PCR and compared with 22Rv1 cells containing the empty vector (22Rv1-VO). EphB4 overexpression resulted in a significant 2.5 fold increase in ITGB8 gene levels, but no significant effect was seen on ITGA3 or ITGA10 expression (Figure 1D). Again, the increase in gene expression of ITGB8 correlated with an increase in protein level (Figure 1E). Together, these data suggest that EPHB4 and ITGB8 are co-regulated in prostate cancer cells. As integrin $\beta 8$ has only one known heterodimer partner, integrin $\alpha \mathrm{V}$ (ITGAV) [10], we also sought to determine whether over-expression of EphB4 increases the expression of this integrin subunit. EPHB4 overexpression in $22 \mathrm{Rv} 1$ cells significantly increased ITGAV gene expression by 1.7 fold (Figure $1 \mathrm{~F}$ ) suggesting an overall increase in the integrin $\alpha v \beta 8$ heterodimer complex.

\section{Knockdown of ITGB8 suppresses migration and invasion in prostate cancer cells}

Integrin $\beta 8$ has been implicated in tumor cell invasiveness [11]. To analyse functional effects of integrin $\beta 8$ in prostate cancer cells, we employed siRNA knockdown. In PC-3 cells, ITGB8 knockdown resulted in a 70\% decrease in ITGB8 mRNA (Figure 2A). PC-3 cells transfected with ITGB8-targeted siRNA showed a significant decrease in in vitro wound healing migration and in Matrigel invasion, when compared with PC-3 cells

Table 2 Top ten significantly down-regulated genes in LNCaP cells after EPHB4 siRNA knockdown

\begin{tabular}{llll}
\hline Fold change & Gene symbol & Description & Function \\
\hline $\mathbf{- 2 9 . 4}$ & ITGB8 & Integrin beta 8 & Cell-cell and cell-extracellular matrix interactions \\
$\mathbf{- 1 0 . 5}$ & LOC100287846 & Patched 1 pseudogene & Uncharacterized \\
$\mathbf{- 1 0 . 5}$ & PROM1 & Prominin 1/CD133 & Transmembrane glycoprotein, stem cell marker \\
$\mathbf{- 1 0 . 3}$ & EIF4E3 & Eukaryotic translation initiation factor 4E family member 3 & Recruits mRNA to ribsosome \\
$\mathbf{- 8 . 8}$ & GBP3 & Guanylate Binding Protein 3 & Binding of guanidine nucleotides \\
$\mathbf{- 8 . 4}$ & DOCK10 & Dedicator of Cytokinesis 10 & Guanine nucleotide exchange factor \\
$\mathbf{- 7 . 9}$ & C14Orf38 & Open reading frame & Uncharacterized \\
$\mathbf{- 6 . 9}$ & TMC7 & Transmembrane Channel-like 7 & Multi-pass membrane protein \\
$\mathbf{- 6 . 8}$ & SASH1 & SAM and SH3 domain Containing 1 & Candidate tumour suppressor in breast cancer \\
$\mathbf{- 5 . 8}$ & FSD2 & Fibronectin type III and SPRY domain containing 2 & Uncharacterized \\
\hline
\end{tabular}


Table 3 Top ten significantly up-regulated genes in LNCaP cells after EPHB4 siRNA knockdown

\begin{tabular}{llll}
\hline Fold change & Gene symbol & Description & Function \\
\hline $\mathbf{5 . 7}$ & MYO6 & Myosin VI & Actin-based motor protein \\
$\mathbf{4 . 8}$ & MMP12 & Matrix-metallopeptidase 12 & Cleavage of extra-cellular matrix \\
$\mathbf{4 . 4}$ & LOC100131726 & HCC-related HCC-C11_V3 & Miscellaneous RNA \\
$\mathbf{4 . 3}$ & TDRD7 & Tudor Domain containing 7 & Component of cytoplasmic RNA granules \\
$\mathbf{4 . 3}$ & OSMR & Oncostatin M receptor & Type I cytokine receptor \\
$\mathbf{4 . 3}$ & APOBEC3H & Apolipoprotein B mRNA editing enzyme, catalytic & Antiretroviral mRNA editing enzyme \\
$\mathbf{4 . 2}$ & PYP11A1 & Cytochrome P450, family 11, subfamily A, polypeptide 1 & Conversion of cholesterol to pregnolone \\
$\mathbf{4 . 2}$ & LRRTM3 & Leucine Rich Repeat Transmembrane Neuronal 3 & Implicated in neuronal disorders \\
$\mathbf{4 . 1}$ & GCOM1 & GRINL1A complex locus 1 & Read-through transcription between the MYZAP \\
& & & (myocardial zonula adherens protein) and POLR2M \\
& TBC1D8B & TBC1 domain family, member 8B & Uncharacterized \\
$\mathbf{3 . 9}$ & &
\end{tabular}

transfected with negative siRNA (Figure 2B \& C). Furthermore, transfection of 22Rv1-VO and 22Rv1-B4 cells with the ITGB8 siRNA resulted in a significant decrease in Matrigel invasion (Figure 2D). There was no significant difference in the adhesion to vitronectin of 22Rv1VO or 22Rv1-B4 cells transfected with ITGB8 siRNA (Figure 2E). Together, these results highlight that integrin $\beta 8$ plays an important role in prostate cancer cell migration and invasion in both endogenous and exogenously over-expressing EphB4 cell models.

\section{Correlation between ITGB8 and EPHB4 expression levels}

To investigate whether there is a correlation between ITGB8 and EPHB4 expression in different prostate cancer cells representing different stages of the disease, several cell lines were subjected to RT-PCR (Figure 3A). All prostate cell lines tested expressed EPHB4 as expected $[5,7]$. ITGB8 was most highly expressed in PC-3 and DU145 cells, followed by BPH-1 and LNCaP cells. The metastatic subclonal line $\mathrm{C} 4-2 \mathrm{~B}$ derived from $\mathrm{LNCaP}$ cells [12] showed no ITGB8 mRNA expression. To further assess whether ITGB8 expression could be indicative of clinical disease progression we surveyed the Oncomine database. Analysis of the prostate cancer progression study conducted by Tomlins et al. [13] revealed that in benign samples both EPHB4 and ITGB8 are expressed at a low level and this increases dramatically and significantly in prostatic intraepithelial neoplasia

\section{Table 4 Significantly enriched gene ontology process in} LNCaP cells after EPHB4 siRNA knockdown

\begin{tabular}{ll}
\hline GO biological process & Genes involved \\
\hline Cell adhesion: & ACHE; CD34; CDH19; CLDN10; CNTN6; COL5A1; \\
GO:0007155 & DLC1; DSC1; DSCAM; FEZ1; FRAS1; ITGA10; ITGA3; \\
& ITGB8; JAM2; LAMA4; MTSS1; NELL2; NLGN3; \\
& NRCAM; PKD2; STAB2; TGFBI; TNC; TNF; VCL \\
\hline
\end{tabular}

(PIN) (ITGB8: 9.5 fold, $\mathrm{p}=1.24 \times 10^{-4}$ and EPHB4 2.9 fold, $\mathrm{p}=0.001)$, the precursor for prostate carcinoma [14]. In carcinoma samples the expression of both genes is elevated, with EPHB4 being significantly up-regulated in comparison to benign tissue samples, but expression of both ITGB8 and EPHB4 is much lower than in PIN tissues. Metastatic samples show expression levels similar to benign tissue for both EPHB4 and ITGB8. These results indicate that $E P H B 4$ and ITGB8 are concurrently up-regulated in PIN and their expression is progressively lowered with disease advancement. This suggests that both genes may play a role in the onset of prostate cancer.

\section{Discussion}

We have recently shown that EphB4 over-expression leads to a more aggressive phenotype in prostate cancer cells [2]. In this study we set out to investigate the gene expression changes that occurred when EPHB4 was knocked down in LNCaP prostate cancer cells that are endogenously over-expressing EphB4. Microarray analysis revealed a set of three integrin subunits $(\alpha 3, \alpha 10$ and $\beta 8$ ) that were de-regulated upon siRNA knockdown of EPHB4 and qRT-PCR validated two of these ( $\alpha 10$ and $\beta 8)$. Over-expression of EPHB4 led to concomitant and parallel changes in expression and protein levels of ITGB8 but not the other two integrins. In keratinocytes, it has been shown that EphB2-induced reverse signaling down-regulated integrin expression, demonstrating that in other cell contexts other Eph receptors also have the ability to influence integrin expression [15]. Integrins are a family of transmembrane receptors which are primarily involved in cell-extracellular matrix (ECM) adhesion as well as cell-cell interactions. By connecting the actin cytoskeleton to the ECM, integrins are able to regulate attachment, cytoskeletal organization, mechano-sensing, 


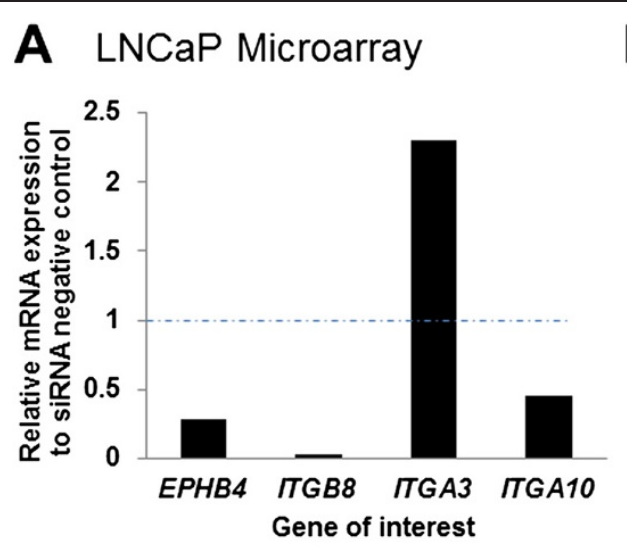

B LNCaP qRT-PCR

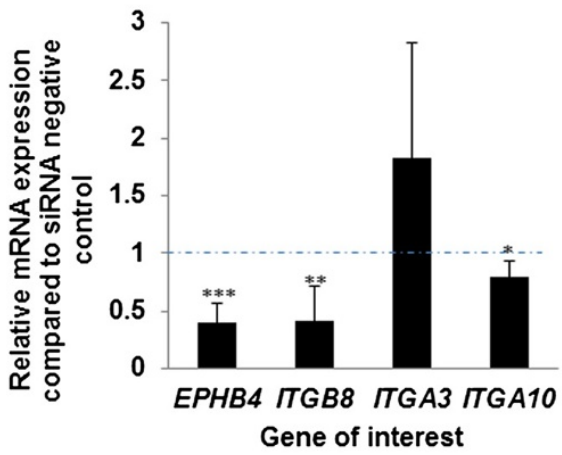

C LNCaP Western

D 22Rv1-B4 qRT-PCR

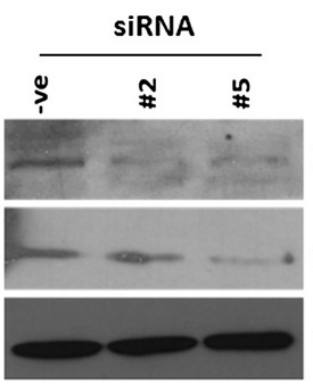

EphB4-120 kDa

Integrin $\beta 8-100 \mathrm{kDa}$

GAPDH - 37 kDa

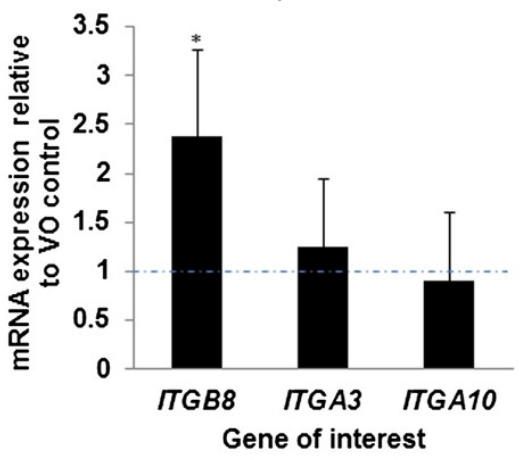

\section{E 22Rv1 Western}

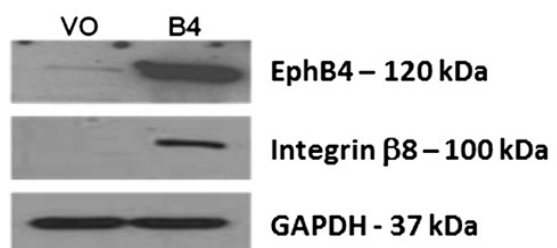

F $\quad 22 R v 1$ qRT-PCR

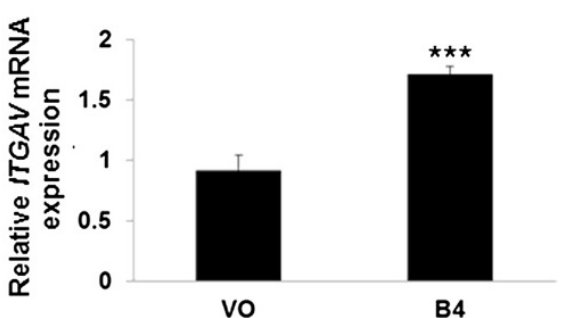

Figure 1 Integrins are significantly de-regulated in response to changing EphB4 levels in prostate cancer cells. A) Relative gene expression of EPHBA, ITGA3, ITGA10 and ITGB8 after siRNA knockdown of EPHB4 in LNCaP cells as identified on the CDNA microarray, compared to control negative siRNA. Dotted line indicates normalized level of negative siRNA control. B) Relative gene expression normalized to GAPDH of EPHB4, ITGA3, ITGA10 and ITGB8 after siRNA knockdown of EPHB4 in LNCaP cells as determined by qRT-PCR (independent experiments from results shown in $\mathbf{A}$ ). Dotted line indicates normalized level of negative siRNA control. C) Western blotting analysis showing integrin $\beta 8$ and EphB4 protein levels in LNCaP cells that have been transfected with two different siRNAs (\#2 and \#5) targeting EPHB4. GAPDH was used to normalize for loading. D) Relative gene expression of ITGA3, ITGA10 and ITGB8, normalized to GAPDH, in stably over-expressing 22Rv1-B4 cells as determined by qRT-PCR. Dotted line indicates normalized level of negative siRNA control. E) Western blotting analysis showing integrin $\beta 8$ and EphB4 protein levels in 22Rv1-B4 over-expressing prostate cancer cells. GAPDH was used as a loading control. F) Relative gene expression of ITGAV in stably over-expressing 22Rv1-B4 compared to VO (vector only) cells as determined by qRT-PCR. QRT-PCR experiments were carried out in triplicate and with three biological replicates. Western blotting experiments were carried out three times and representative cropped blots are shown. Graphs are presented with \pm SD. ${ }^{* *} p<0.005$, ${ }^{* *} p<0.01,{ }^{*} p<0.05$.

migration, proliferation, differentiation and cell survival [16]. Through their several roles, integrins have been found to be involved in a range of pathological processes including tumor angiogenesis and metastasis $[17,18]$.

There is evidence that Eph/ephrin signaling can influence integrin clustering and in some cases, inhibit integrin downstream signaling $[19,20]$. Furthermore, ephrin-A1 and
EphA2 have both been shown to co-localize with integrin $\alpha 3$ [20-22]. In breast cancer cell lines, EphB4 exogenous overexpression reduces integrin $\beta 1$ expression resulting in increased migration in a ligand-independent manner [23]. In the current study, validation experiments confirmed that ITGB8 was significantly down-regulated when EPHB4 was knocked down and moreover, was also up-regulated when 

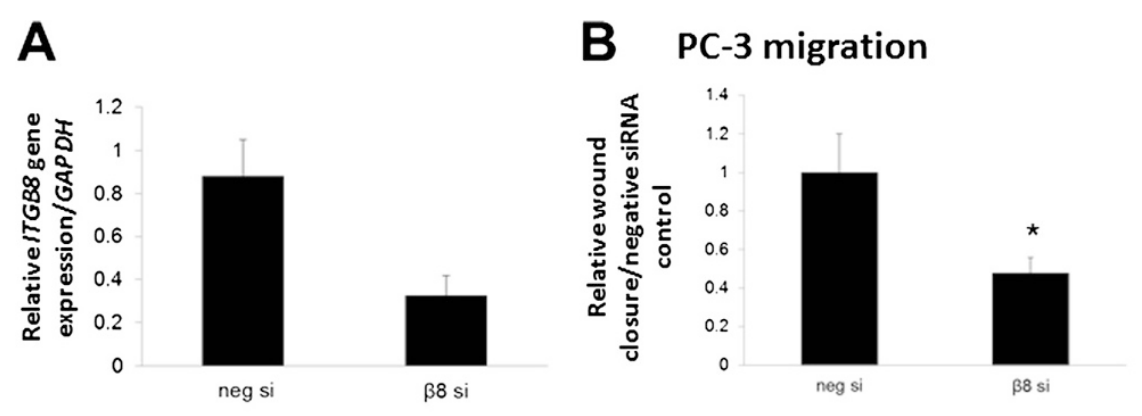

C PC-3 invasion

D 22Rv1 invasion
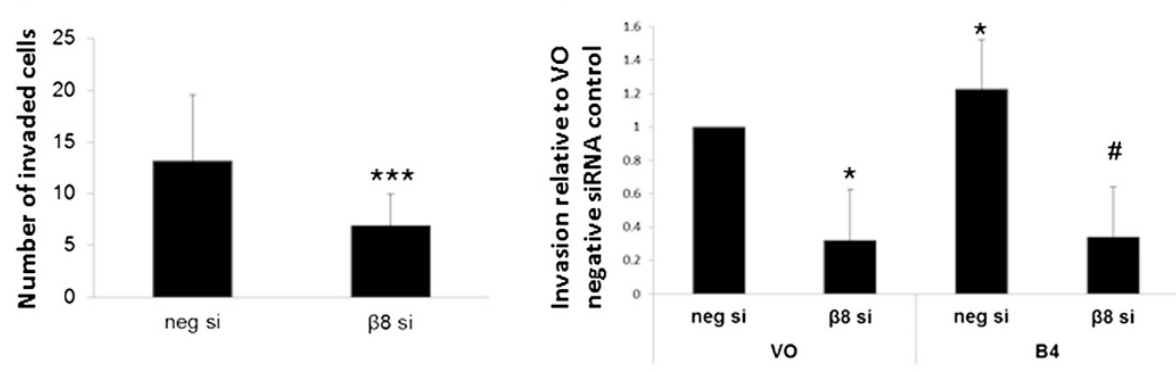

\section{E 22Rv1 adhesion}

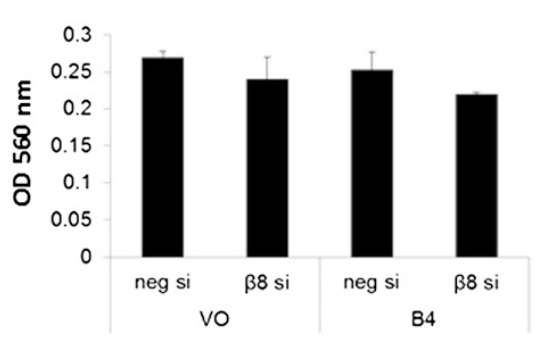

Figure 2 Knockdown of ITGB8 results in reduced metastatic potential in prostate cancer cells. A) Quantitative real-time PCR was carried out to determine knockdown levels of siRNA against ITGB8. 100 nM negative non-silencing or ITGB8 targeting siRNA were transiently transfected into PC-3 cells. RNA was isolated $48 \mathrm{~h}$ after transfection, transcribed into CDNA and analyzed for gene expression. ITGB8 expression is reduced by approximately 60-70\%. B) PC-3 cells were transiently transfected with $100 \mathrm{nM}$ negative non-silencing (neg si) or ITGB8 targeting siRNA ( $\beta 8$ si) and $24 \mathrm{~h}$ later a scratch wound was applied using the IncuCyte (Essen Bioscience) system and migration was monitored for a further $24 \mathrm{~h}$. Cell migration was reduced following knockdown of ITGB8. C) PC-3 cells were transiently transfected with 100 nM negative non-silencing (neg si) or ITGB8 targeting siRNA ( $\beta 8$ si) and subjected to a Matrigel transwell invasion assay. After $22 \mathrm{~h}$ of incubation, invaded cells were stained and counted. Cell invasion was reduced following knockdown of ITGB8. D) 22Rv1-VO (vector only) or 22Rv1-B4 (EphB4 over-expressing) cells were transiently transfected with 100 nM siRNA against ITGB8 ( $B 8$ si) or negative non-silencing siRNA (neg si). An invasion assay was carried out using the Matrigel invasion system and cells were allowed to invade for $22 \mathrm{~h}$. Cells containing the ITGB8 siRNA showed significantly reduced ability to invade. E) 22Rv1-VO (vector only) or 22Rv1-B4 (EphB4 over-expressing) cells were transiently transfected with siRNA against ITGB8 ( $\beta 8$ si) or negative non-silencing siRNA (neg si) and subjected to an adhesion assay to vitronectin. No significant changes were seen. $n=3 * p<0.01$ vs VO negative; $p<0.001$ vs B4 negative.

EPHB4 was over-expressed in prostate cancer cells. This suggests that EPHB4 and ITGB8 are potentially transcriptionally co-regulated. In terms of transcriptional regulation, the ITGB8 promotor contains SP and CRE binding motifs and is regulated by SP1, SP3 and an AP-1 complex [24]. EPHB4 on the other hand has been shown to be transcriptionally regulated by HoxA9 in endothelial cells [25], but no information is available about the transcription factors involved in regulating expression of EPHB4 in cancer cells. It would be interesting to investigate whether the transcription factors of the SP family can also influence EPHB4 expression in prostate cancer and thus be responsible for coregulating ITGB8 and EPHB4.

Although only limited information is available about the role of integrin $\beta 8$ in cancer it has been identified as up-regulated in several cancers including head and neck cancer, hepatocellular carcinoma, some ovarian cancer and melanoma cell lines as well as primary non-small lung cancer samples and brain metastases from several 


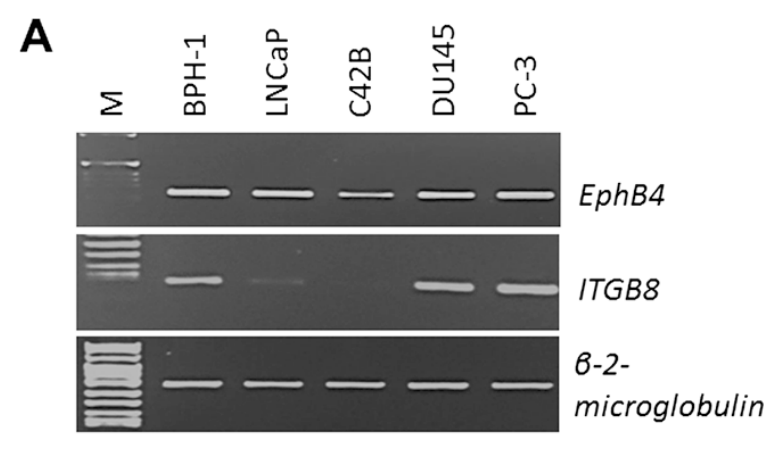

B

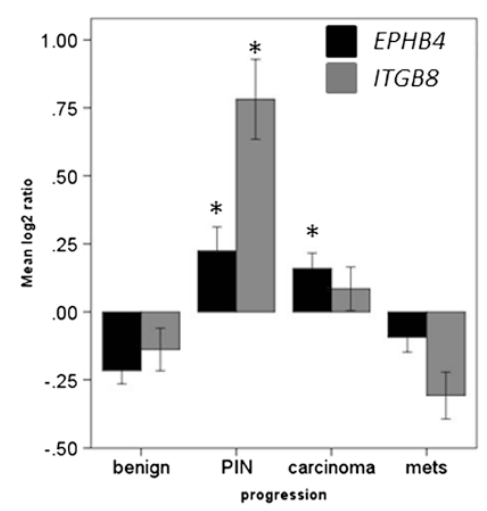

Figure 3 Integrin expression levels across disease progression. A) CDNA from several different prostate-derived cell lines was analyzed using semi-quantitative RT-PCR. GAPDH amplification was used as a loading control. B) Gene expression omnibus dataset GDS3289 investigating prostate cancer progression in LCM-captured clinical samples was interrogated for EPHB4 and ITGB8 expression using the Oncomine clinical database (www.oncomine.org). Both genes are significantly elevated in prostatic intraepithelial neoplasia (PIN) and EPHB4 is significantly upregulated in carcinoma samples compared to benign. ${ }^{*} \mathrm{p}<0.001$, mets $=$ metastatic disease.

epithelial cancers [26-28]. Furthermore, ITGB8 has been identified as a member of a six-gene expression signature biomarker predicting lung metastasis from breast cancer [29]. In glioblastoma specimens, there appears to be a correlation between low integrin $\beta 8$ expression and highly angiogenic, but poorly invasive tumors; as well as high integrin $\beta 8$ expression in low angiogenic and highly invasive tumors (17). This implies that integrin $\beta 8$ is involved in differential control of angiogenesis versus tumor cell invasion in glioblastomas. On the other hand, integrin $\beta 8$ has been shown to be growth inhibitory in lung cancer cells, yet it is also playing a fundamental role in lung cancer metastasis indicating that this integrin functions in a complex manner in cancer presumably depending on tissue and progression context [30-32]. As the expression of integrin $\beta 8$ has been found to be increased in metastases from various tumors, including breast and lung, we speculated that integrin $\beta 8$ could also play a role in prostate cancer cell migration and invasion and we show that silencing of ITGB8 reduces cell motility $[28,29]$. In highly invasive glioblastoma tumors, high levels of integrin $\beta 8$ cooperate with Rho proteins to drive invasion [11]. Eph receptors are also able to activate the Rho pathway to regulate cancer cell migration [33] and thus it is possible that EphB4 and integrin $\beta 8$ work cooperatively to control cell motility.

By interrogating the Oncomine database we have identified ITGB8 as being up-regulated in PIN, the precursor to prostate cancer which is puzzling considering its role in metastases. It is possible that integrin $\beta 8$ is able to impact on different stages of tumor development in different cell populations. In established prostate cancer cell lines we demonstrate that integrin $\beta 8$ plays a vital role in migration and invasion. These results are in agreement with previous reports describing similar findings in lung cancer and glioblastoma [11,31].

Integrin $\beta 8$ has been shown to only heterodimerise with the integrin $\alpha \mathrm{v}$ subunit and this heterodimer binds to vitronectin [10]. We show here that the $\alpha v$ subunit expression also increases in stably EphB4 overexpressing prostate cancer cells, but no effect on adhesion to vitronectin was seen. Interestingly, transforming growth factor $\beta$ (TGF- $\beta$ ) has been identified as the only physiologically relevant ligand for integrin $\alpha v \beta 8$ and activation of matrix-bound latent TGF- $\beta$ by integrin $\alpha v \beta 8$ results in activation of TGF- $\beta$ signaling and remodeling of human airway fibroblasts [34]. TGF- $\beta$ is a well-known cytokine with a variety of physiological functions such as proliferation, differentiation and immune response and in addition it plays a major role in cancer progression by inducing epithelial-to-mesenchymal transition (EMT) in prostate cancer and promoting metastasis to the bone, the final step in prostate cancer progression [35,36]. In our array data TGFB1 was also found to be significantly upregulated by 2 -fold (data not shown). This could indicate that EPHB4, ITGB8 and TGFB1 are intrinsically regulated in prostate cancer cells, therefore contributing to cancer progression and metastasis through the process of EMT. However, expression analysis of the EMT markers E-cadherin and Snail did not show any significant changes in PC-3 cells transfected with ITGB8 siRNA (data not shown). Changes in E-cadherin and Snail expression have been reported in lung cancer cells where ITGB8 was silenced [31].

Cilengitide, a selective antagonist of $\alpha v \beta 3$ and $v \beta 5$ integrins, is currently in clinical trials for a variety of solid tumors, but so far the results are modest [37-39]. In prostate cancer, no clinical effect was seen [40]. Targeting the $\alpha v \beta 8$ integrin in combination with EphB4 targeted therapies may represent a future avenue for prostate cancer therapy. 


\section{Conclusions}

Alteration of EphB4 levels (through knockdown or overexpression) concurrently, and in a similar manner, alters the levels of integrin $\beta 8$. The high level of expression of both EPHB4 and ITGB8 in clinical PIN samples suggests that their increased expression is an early event in the development of prostate cancer. This also identifies a new mechanism for EphB4 function in prostate cancer through the regulation of ITGB8, which our results show can contribute to prostate cancer cell motility. Targeting both EphB4 and integrin $\beta 8$ may provide new options for treating prostate cancer.

\section{Additional file}

Additional file 1: Figure S1. Microarray analysis of de-regulated genes after siRNA knockdown of EPHB4 in LNCaP prostate cancer cells. Volcano plot representing up-and down-regulated genes (log2 scale, fold change) comparing non-silencing siRNA samples $(n=3)$ to EPHB4 knockdown samples $(n=4)$. The analysis was carried out using Genespring GX11 (Agilent Technologies).

\section{Competing interests}

The authors declare that they have no competing interests.

\section{Authors' contributions}

IMW conceived the study, coordinated it and carried out transwell migration and invasion assays, real-time PCR, ONCOMINE database analysis as well as statistical analysis and wrote the manuscript. BC carried out real-time PCR and western blotting. MM carried out the wound migration assay. AR hybridized the samples to the microarray and helped with the data analysis. CC provided the microarrays and helped coordinate the microarray study. ACH and SAS helped in planning of the study and drafted the manuscript. All authors read and approved the final manuscript.

\section{Acknowledgements}

This work was supported by an Early Career Researcher grant awarded to IM-W by the Institute of Health and Biomedical Innovation and grants to ACH and S-AS from the Australian Prostate Cancer Research Centre-Queensland and Queensland University of Technology.

Received: 1 October 2014 Accepted: 5 March 2015

Published online: 22 March 2015

\section{References}

1. Pasquale EB. Eph receptor signalling casts a wide net on cell behaviour. Nat Rev Mol Cell Biol. 2005;6:462-75.

2. Rutkowski R, Mertens-Walker I, Lisle JE, Herington AC, Stephenson SA. Evidence for a dual function of EphB4 as tumor promoter and suppressor regulated by the absence or presence of the ephrin-B2 ligand. Int I Cancer. 2012;131:E614-24.

3. Dopeso H, Mateo-Lozano S, Mazzolini R, Rodrigues P, Lagares-Tena L, Ceron J, et al. The receptor tyrosine kinase EPHB4 has tumor suppressor activities in intestinal tumorigenesis. Cancer Res. 2009;69:7430-8.

4. Kumar SR, Singh J, Xia G, Krasnoperov V, Hassanieh L, Ley EJ, et al. Receptor tyrosine kinase EphB4 is a survival factor in breast cancer. Am J Pathol. 2006:169:279-93.

5. Xia G, Kumar SR, Masood R, Zhu S, Reddy R, Krasnoperov V, et al. EphB4 expression and biological significance in prostate cancer. Cancer Res. 2005;65:4623-32.

6. Noren NK, Foos G, Hauser CA, Pasquale EB. The EphB4 receptor suppresses breast cancer cell tumorigenicity through an Abl-Crk pathway. Nat Cell Biol. 2006:8:815-25.

7. Lee YC, Perren JR, Douglas EL, Raynor MP, Bartley MA, Bardy PG, et al. Investigation of the expression of the EphB4 receptor tyrosine kinase in prostate carcinoma. BMC cancer. 2005;5:119.
8. Sieh S, Taubenberger AV, Rizzi SC, Sadowski M, Lehman ML, Rockstroh A, et al. Phenotypic characterization of prostate cancer LNCaP cells cultured within a bioengineered microenvironment. PloS One. 2012;7:e40217.

9. Stephenson SA, Slomka S, Douglas EL, Hewett PJ, Hardingham JE. Receptor protein tyrosine kinase EphB4 is up-regulated in colon cancer. BMC Mol Biol. 2001;2:15.

10. Nishimura SL, Sheppard D, Pytela R. Integrin alpha $v$ beta 8 . Interaction with vitronectin and functional divergence of the beta 8 cytoplasmic domain. J Biol Chem. 1994;269:28708-15.

11. Reyes SB, Narayanan AS, Lee HS, Tchaicha JH, Aldape KD, Lang FF, et al. Alphavbeta8 integrin interacts with RhoGDI1 to regulate Rac1 and Cdc42 activation and drive glioblastoma cell invasion. Mol Biol Cell. 2013;24:474-82

12. Thalmann GN, Anezinis PE, Chang SM, Zhau HE, Kim EE, Hopwood VL, et al. Androgen-independent cancer progression and bone metastasis in the LNCaP model of human prostate cancer. Cancer Res. 1994;54:2577-81.

13. Tomlins SA, Mehra R, Rhodes DR, Cao X, Wang L, Dhanasekaran SM, et al. Integrative molecular concept modeling of prostate cancer progression. Nat Genet. 2007;39:41-51.

14. Bostwick DG, Qian J. High-grade prostatic intraepithelial neoplasia. Mod Pathol. 2004;17:360-79.

15. Walsh R, Blumenberg M. Eph-2B, acting as an extracellular ligand, induces differentiation markers in epidermal keratinocytes. J Cell Physiol. 2012;227:2330-40

16. Morse EM, Brahme NN, Calderwood DA. Integrin cytoplasmic tail interactions. Biochemistry. 2014;53:810-20.

17. Goodman SL, Picard M. Integrins as therapeutic targets. Trends Pharmacol Sci. 2012;33:405-12.

18. Weis SM, Cheresh DA. Tumor angiogenesis: molecular pathways and therapeutic targets. Nat Med. 2011;17:1359-70.

19. Jülich D, Mould AP, Koper E, Holley SA. Control of extracellular matrix assembly along tissue boundaries via integrin and Eph/Ephrin signaling. Development. 2009;136:2913-21.

20. Bourgin C, Murai KK, Richter M, Pasquale EB. The EphA4 receptor regulates dendritic spine remodeling by affecting beta1-integrin signaling pathways. J Cell Biol. 2007;178:1295-307.

21. Zou JX, Wang B, Kalo MS, Zisch AH, Pasquale EB, Ruoslahti E. An Eph receptor regulates integrin activity through R-Ras. Proc Natl Acad Sci U S A. 1999;96:13813-8

22. Makarov A, Ylivinkka I, Nyman TA, Hyytiainen M, Keski-Oja J. Ephrin-As, Eph receptors and integrin alpha3 interact and colocalise at membrane protrusions of U251MG glioblastoma cells. Cell Biol Int. 2013;37:1090-88.

23. Noren NK, Yang NY, Silldorff M, Mutyala R, Pasquale EB. Ephrin-independent regulation of cell substrate adhesion by the EphB4 receptor. Biochem J. 2009:422:433-42.

24. Markovics JA, Araya J, Cambier S, Jablons D, Hill A, Wolters PJ, et al. Transcription of the transforming growth factor beta activating integrin beta8 subunit is regulated by SP3, AP-1, and the p38 pathway. J Biol Chem. 2010;285:24695-706.

25. Bruhl T, Urbich C, Aicher D, Acker-Palmer A, Zeiher AM, Dimmeler S Homeobox A9 transcriptionally regulates the EphB4 receptor to modulate endothelial cell migration and tube formation. Circ Res. 2004;94:743-51.

26. Liu LX, Jiang HC, Liu ZH, Zhou J, Zhang WH, Zhu AL, et al. Integrin gene expression profiles of human hepatocellular carcinoma. World J Gastroenterol. 2002:8:631-7.

27. Goodman SL, Grote HJ, Wilm C. Matched rabbit monoclonal antibodies against alphav-series integrins reveal a novel alphavbeta3-LIBS epitope, and permit routine staining of archival paraffin samples of human tumors. Biol Open. 2012;1:329-40

28. Vogetseder A, Thies S, Ingold B, Roth P, Weller M, Schraml P, et al. AlphavIntegrin isoform expression in primary human tumors and brain metastases. Int J Cancer. 2013;133:2362-71.

29. Landemaine T, Jackson A, Bellahcene A, Rucci N, Sin S, Abad BM, et al. A six-gene signature predicting breast cancer lung metastasis. Cancer Res. 2008;68:6092-9.

30. Cambier S, Mu D-Z, O'Connell D, Boylen K, Travis W, Liu W-H, et al. A Role for the Integrin av $\beta 8$ in the negative regulation of epithelial cell growth. Cancer Res. 2000;60:7084-93.

31. Xu Z, Wu R. Alteration in metastasis potential and gene expression in human lung cancer cell lines by ITGB8 silencing. Anat Rec (Hoboken). 2012;295:1446-54 
32. Fang L, Deng Z, Shatseva T, Yang J, Peng C, Du WW, et al. MicroRNA miR-93 promotes tumor growth and angiogenesis by targeting integrin-beta8. Oncogene. 2011;30:806-21.

33. Astin JW, Batson J, Kadir S, Charlet J, Persad RA, Gillatt D, et al. Competition amongst Eph receptors regulates contact inhibition of locomotion and invasiveness in prostate cancer cells. Nat Cell Biol. 2010;12:1194-204.

34. Nishimura SL. Integrin-mediated transforming growth factor-beta activation, a potential therapeutic target in fibrogenic disorders. Am J Pathol. 2009;175:1362-70.

35. Juarez P, Guise TA. TGF-beta in cancer and bone: implications for treatment of bone metastases. Bone. 2011;48:23-9.

36. Jones $\mathrm{E}, \mathrm{Pu} \mathrm{H}$, Kyprianou N. Targeting TGF-beta in prostate cancer: therapeutic possibilities during tumor progression. Expert Opin The Targets. 2009;13:227-34

37. Eisele G, Wick A, Eisele AC, Clement PM, Tonn J, Tabatabai G, et al. Cilengitide treatment of newly diagnosed glioblastoma patients does not alter patterns of progression. J Neurooncol. 2014;117:141-5.

38. Vermorken JB, Peyrade F, Krauss J, Mesia R, Remenar E, Gauler TC, et al. Cisplatin, 5-fluorouracil, and cetuximab (PFE) with or without cilengitide in recurrent/metastatic squamous cell carcinoma of the head and neck: results of the randomized phase I/II ADVANTAGE trial (phase II part). Ann Oncol. 2014;25:682-8.

39. Manegold C, Vansteenkiste J, Cardenal F, Schuette W, Woll PJ, Ulsperger E, et al. Randomized phase II study of three doses of the integrin inhibitor cilengitide versus docetaxel as second-line treatment for patients with advanced non-small-cell lung cancer. Invest New Drugs. 2013;31:175-82.

40. Alva A, Slovin S, Daignault S, Carducci M, Dipaola R, Pienta K, et al. Phase II study of cilengitide (EMD 121974, NSC 707544) in patients with nonmetastatic castration resistant prostate cancer, NCl-6735. A study by the DOD/PCF prostate cancer clinical trials consortium. Invest New Drugs. 2012;30:749-57.

\section{Submit your next manuscript to BioMed Central and take full advantage of:}

- Convenient online submission

- Thorough peer review

- No space constraints or color figure charges

- Immediate publication on acceptance

- Inclusion in PubMed, CAS, Scopus and Google Scholar

- Research which is freely available for redistribution 